\title{
Ovarian Mesonephric-like Adenocarcinoma With Multifocal Microscopic Involvement of the Fimbrial Surface: Potential for Misdiagnosis of Tubal Intraepithelial Metastasis as Serous Tubal Intraepithelial Carcinoma Associated With Ovarian High-grade Serous Carcinoma
}

\author{
HYUNJIN KIM ${ }^{1}$, GO EUN BAE ${ }^{2}$, YOON YANG JUNG ${ }^{3}$ and HYUN-SOO KIM ${ }^{1}$ \\ ${ }^{1}$ Department of Pathology and Translational Genomics, Samsung Medical Center, \\ Sungkyunkwan University School of Medicine, Seoul, Republic of Korea; \\ ${ }^{2}$ Department of Pathology, Chungnam National University School of Medicine, Daejeon, Republic of Korea; \\ ${ }^{3}$ Department of Pathology, Myongji Hospital, \\ Hanyang University College of Medicine, Goyang, Republic of Korea
}

\begin{abstract}
Background/Aim: We describe a rare case of ovarian mesonephric-like adenocarcinoma (MLA) involving the fimbria and mimicking serous tubal intraepithelial carcinoma (STIC). Case Report: A 47-year-old woman presented with a 4.4-cm left ovarian mass. Histologically, the ovarian tumor showed papillary and solid architecture, severe nuclear pleomorphism, and increased mitotic activity. Some microscopic foci where the tumor cells spread horizontally along the fimbrial surface epithelium were noted, compatible with STIC. We initially considered the ovarian tumor to be high-grade serous carcinoma accompanied by a fimbrial STIC. However, immunostaining revealed nuclear immunoreactivity for paired box 2 and GATA-binding protein 3, but lacked expression of Wilms tumor 1. A thorough slide review and additional immunostaining revealed architectural diversity, densely eosinophilic intraluminal secretions, and lack of
\end{abstract}

This article is freely accessible online.

Correspondence to: Hyun-Soo Kim, Department of Pathology and Translational Genomics, Samsung Medical Center, Sungkyunkwan University School of Medicine, 81, Irwon-ro, Gangnam-gu, Seoul 06351, Republic of Korea. Tel: +82 234101243, Fax: +82 234142831, e-mail: hyun-soo.kim@samsung.com; Yoon Yang Jung, Department of Pathology, Myongji Hospital, Hanyang University College of Medicine, 14beon-gil, Hwasu-ro, Goyang-si, Gyeonggido 10475, Republic of Korea. Tel: +82 318107101, Fax: +82 318107109, e-mail: jy@mjh.or.kr

Key Words: Ovary, mesonephric-like adenocarcinoma, tubal intraepithelial metastasis, serous tubal intraepithelial carcinoma, high-grade serous carcinoma. hormone receptor expression, supporting the diagnosis of MLA. Conclusion: Microscopic intraepithelial metastases of the MLA to the fimbria mimic STIC. We recommend ancillary tests, such as immunostaining, in patients with ovarian tumors whenever possible, particularly for those with differential diagnosis of MLA and high-grade serous carcinoma.

Ovarian carcinoma is the deadliest gynecological malignancy, accounting for more than 14,000 deaths each year (1). Highgrade serous carcinoma (HGSC) is the most prevalent and aggressive subtype of ovarian carcinoma, accounting for $70 \%$ of ovarian carcinoma-related deaths. The fallopian tube has recently emerged as an important site of origin not only for tubo-ovarian HGSC in patients with germline mutation of breast cancer 1 (BRCA1) or BRCA2, but also as a source of peritoneal HGSC (2). A thorough histological examination of resected ovaries and fallopian tubes has led researchers to focus on investigating the carcinogenesis of tubo-ovarian HGSC from the fallopian tube. Increasing evidence suggests that the distal part of the fallopian tube, particularly the fimbria, is the origin of tubo-ovarian and peritoneal HGSCs, and serous tubal intraepithelial carcinoma (STIC) is the precursor lesion (3). STIC is morphologically characterized by the proliferation of non-ciliated epithelium, showing stratification, loss of polarity, severe nuclear pleomorphism, conspicuous nucleoli, and increased mitotic activity $(1,4)$. In addition to these histological criteria, more than $90 \%$ of STICs harbor mutations of tumor protein 53 (TP53), resulting in aberrant expression of p53 on immunostaining. Therefore, the lesions typically demonstrate either p53 overexpression (indicating missense mutations) or complete absence of p53 staining (indicating nonsense or frameshift mutations) (5). 
Mesonephric adenocarcinoma (MA) is a rare malignant tumor thought to arise from the embryonic remnants of the mesonephric tubules and ducts, and comprises less than $1 \%$ of all gynecological malignancies (6). MA exhibits a variety of histological growth patterns, including tubular, ductal, papillary, solid, spindle, retiform, sex cord-like, and comedo necrosis-like (7-13). The small tubular and ductal structures contain eosinophilic intraluminal secretions. MA typically arises in the uterine cervix or vagina, but several cases arising in the upper female genital tract have also been reported $(14,15)$. Even though they share the same histological features and immunophenotypes with MA, their association with mesonephric remnants has not been firmly established $(6,14)$. Within this context, MA of the uterine corpus or adnexa is referred to in the literature as a mesonephric-like adenocarcinoma (MLA).

MLA of the ovary is a rare but distinct subtype of ovarian carcinoma (15). It has been newly included in the fifth edition of the World Health Organization Classification of Female Genital Tumors (5). We recently encountered a rare case of ovarian MLA mimicking HGSC accompanied by multifocal STICs involving the fimbrial surface. The presence of fimbrial STIC-like lesions, together with dominant papillary and solid growth patterns and high-grade nuclear atypia of the ovarian tumor, led to an initial diagnosis of ovarian HGSC. However, the unexpected immunostaining results prompted the re-examination of slides, additional immunostaining, and targeted sequencing analyses. This report aimed to provide a thorough clinicopathological description of ovarian MLA showing multifocal tubal intraepithelial metastases (IEMs), as well as its immunophenotype and genetic features. Pathologists play an important role in making appropriate therapeutic plans for patients by accurately determining the histological subtype. Our comprehensive clinicopathological and molecular analyses can help improve the understanding of this rare condition and assist pathologists in making an accurate diagnosis.

\section{Case Report}

The Institutional Review Board (Samsung Medical Center, Seoul, Republic of Korea) granted permission for this study (2021-06-137) to be published on the condition that no patient-identifiable data should be included. Written informed consent for publication was not required by the Institutional Review Board as this study did not include data that could potentially or clearly identify the patient.

A 47-year-old woman presented with a pelvic mass detected on a computed tomography scan that was performed in a different hospital. She underwent total hysterectomy for uterine adenomyosis 4 years ago. Physical examination revealed a non-mobile, non-tender, left pelvic mass.
Magnetic resonance imaging revealed a multilobulated, mixed solid and cystic mass in the left ovary measuring 4.4 $\mathrm{cm}$, which appeared to invade the left proximal ureter, causing hydronephrosis of the left kidney. No definite seeding nodule was observed in the abdominal and pelvic peritoneum but a small amount of ascites with mild diffuse peritoneal thickening was noted. A few enlarged lymph nodes, measuring up to $5.6 \mathrm{~cm}$, were detected in the left para-aortic and retrocrural areas, all of which raised suspicion of metastatic lymphadenopathy. Consistent with these findings, positron-emission tomography/computed tomography images revealed an intensely hypermetabolic mass in the left ovary and left para-aortic areas. Hypermetabolic lymph nodes were also noted in the bilateral retrocrural, left mediastinal, and bilateral supraclavicular areas. The preoperative serum cancer antigen 125 level was elevated $(206.7 \mathrm{U} / \mathrm{ml})$. As the preoperative clinical impression was ovarian carcinoma, primary debulking surgery, including left salpingo-oophorectomy, bilateral pelvic lymphadenectomy, para-aortic lymphadenectomy, total omentectomy, peritonectomy, and intraoperative peritoneal washing cytology, were performed.

Histologically, the ovarian mass presented as a wellcircumscribed, partially encapsulated, round, solid tumor (Figure 1A). The tumor consisted of a central irregularshaped area undergoing hyaline and hydropic degeneration, and a peripheral hypercellular zone showing tumor cell sheets and haphazardly infiltrating the fibrous stroma. Diverse growth patterns were observed, including irregularly-shaped solid masses of tumor cells with slit-like spaces, papillary and micropapillary structures, and large, cribriform, and pseudoendometrioid glands (Figure 1B). Distorted, angulated glands also destructively infiltrated the myxoid stroma (Figure 1C). Slit-like glandular spaces contained inflammatory cells and necrotic debris (Figure 1D). The amount of intervening stroma was minimal. Most of the tumor cells demonstrated high-grade nuclear atypia, including enlargement, hyperchromasia, severe pleomorphism, increased mitotic activity (Figure 1E), conspicuous nucleoli, and occasional atypical mitoses (Figure 1F). These histological features were consistent with those of ovarian HGSC. In addition, the tip and edge of the fimbrial plica showed multiple microscopic foci, showing a significant increase in epithelial thickness compared with the adjacent epithelium (Figure 2A). The thickened epithelium exhibited irregular stacking of enlarged and hyperchromatic nuclei, loss of polarity, and atypical mitoses, while the adjacent normal tubal epithelium was arranged as a single layer of ciliated low-columnar cells with bland-appearing nuclei (Figure 2B). Transition points were evident between the normal and neoplastic epithelium. The subepithelial stroma was unremarkable, without evidence of invasion. We also observed architectural complexity with small, detached, and 

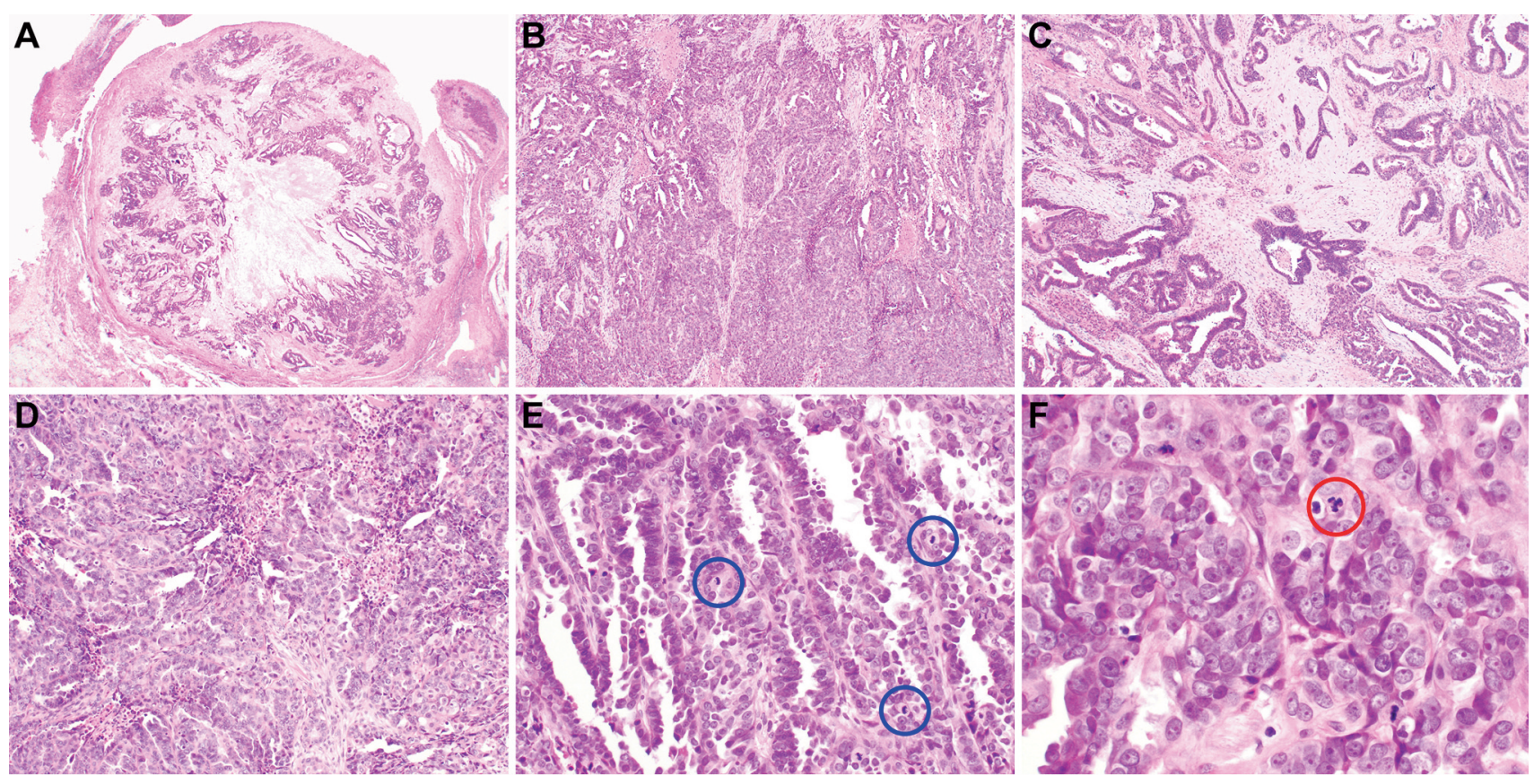

Figure 1. Histological features of the ovarian lesion observed in the initial microscopic examination using hematoxylin and eosin. A: A wellcircumscribed, partially encapsulated, round mass consisted of haphazardly infiltrating sheets and nests of tumor cells. Variable-sized, irregularshaped glands were also noted. The intervening fibrous and myxoid stroma was evident. A large, irregular area of hyaline and hydropic degenerative changes was observed in the center. B: The tumor showed a variety of architectural patterns including irregular-shaped solid masses of tumor cells with slit-like spaces, papillary and micropapillary structures, pseudoendometrioid and cribriform glands, and small tubules. C: Angulated and dilated glands destructively infiltrated the myxoid stroma. D: This hypercellular area showed solid sheets and nests of tumor cells with occasional slit-like spaces and minimal intervening stroma. E: Most of the tumor cells demonstrated high-grade cytological atypia including nuclear enlargement and hyperchromasia, severe nuclear pleomorphism, and frequent mitotic figures (blue circles). F: High-power magnification revealed readily identifiable conspicuous nucleoli, severe nuclear pleomorphism, and an atypical mitotic figure (red circle). Original magnification: A: 10x; $B, C: 40 x ; D: 100 x ; E: 200 x ;$ and F: 400x.

micropapillary clusters, as well as fusion of the micropapillae and focal cribriform appearance (Figure 2C). The presence of architectural abnormalities and a degree of nuclear atypia similar to that of the ovarian tumor led to the diagnosis of STIC. The tumor cells involving the ovarian surface had a similar growth pattern - a partial replacement of the ovarian surface mesothelium by the neoplastic epithelium with abrupt transition points (Figure $2 \mathrm{E}$ and $\mathrm{F}$ ). Several microscopic foci of peritoneal metastases appeared as well-circumscribed tissue plaques, giving the impression that these were plastered on the peritoneal surface (Figure $2 \mathrm{G}$ and $\mathrm{I}$ ). Their cytological features were the same as those of fimbrial lesions. Coexisting ovarian invasive carcinoma, tubal intraepithelial carcinoma horizontally spreading on the fimbrial surface, and multifocal peritoneal involvement were highly suggestive of HGSC accompanied by STIC.

Immunostaining was performed to confirm our diagnosis, as previously described (16-28). A panel of antibodies against Wilms tumor 1 (WT1), p53, estrogen receptor (ER), paired box 8 (PAX8), PAX2, GATA-binding protein 3 (GATA3), and
P16 was used. Contrary to our expectations, the ovarian tumor showed a complete lack of WT1 expression (Figure $3 \mathrm{~A})$ and patchy nuclear p53 immunoreactivity with weak-tomoderate staining intensity (wild-type p53 expression pattern; Figure 3B), neither result was compatible with HGSC. ER expression was not observed (Figure 3C). Instead, cells were diffusely positive for PAX8, PAX2, and GATA3 (Figure 3D), with moderate to strong staining intensity. In all foci considered initially as STICs, we observed the same immunophenotype as that of the ovarian tumor. The tumor cells spreading along the epithelium of the fimbrial surface expressed PAX2 (Figure 3E) and GATA3, but not WT1 (Figure $3 \mathrm{~F}$ and $\mathrm{G}$ ). The fimbrial tumor cells displayed patchy P16 expression (Figure 3H) and wild-type p53 immunoreactivity pattern (Figure 3I and J).

Due to the unexpected immunostaining results, all hematoxylin and eosin-stained slides were thoroughly reviewed. Several foci demonstrated compactly aggregated small tubular structures in areas initially considered as those showing solid architecture. Moreover, densely eosinophilic 

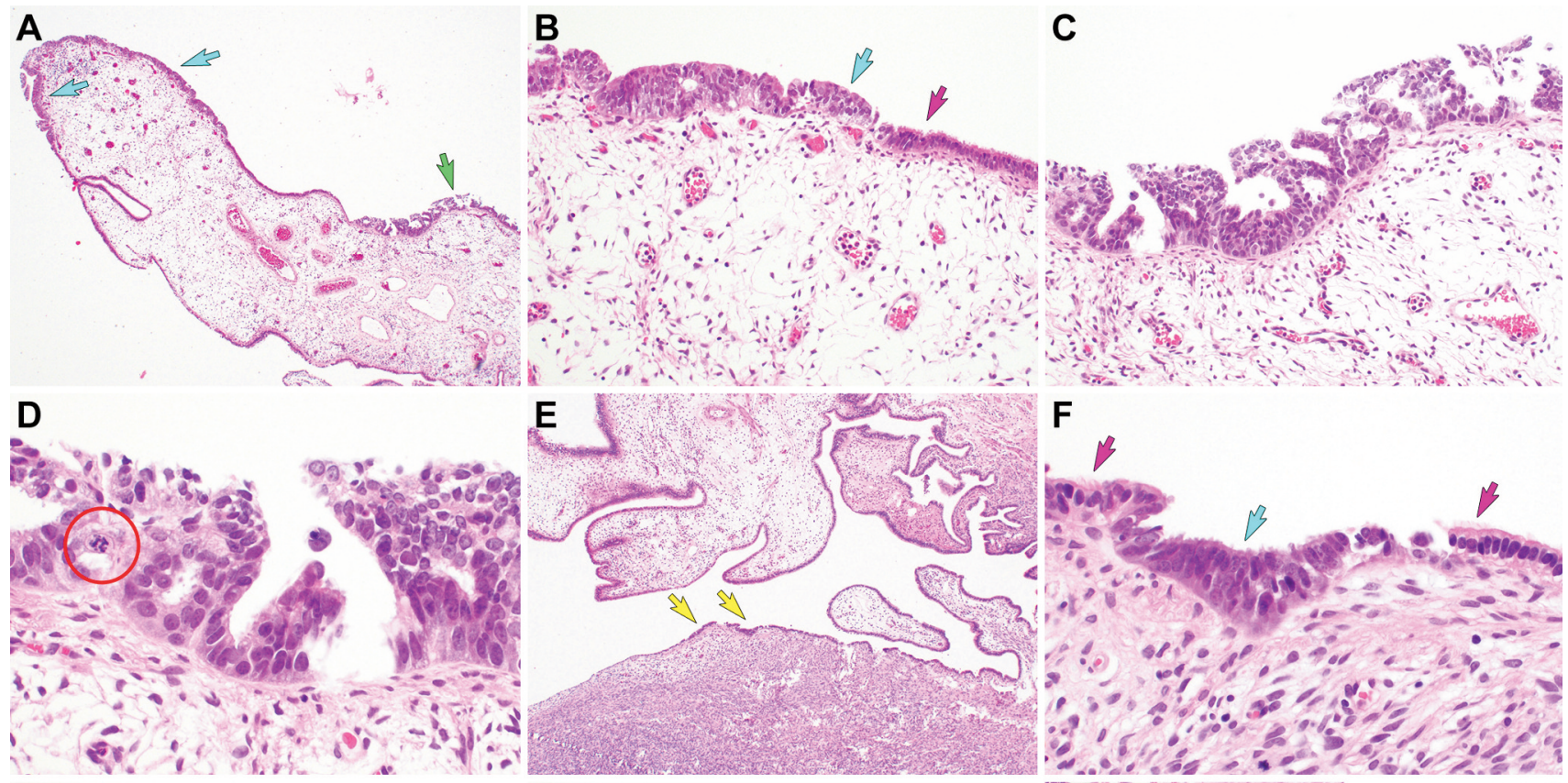

G
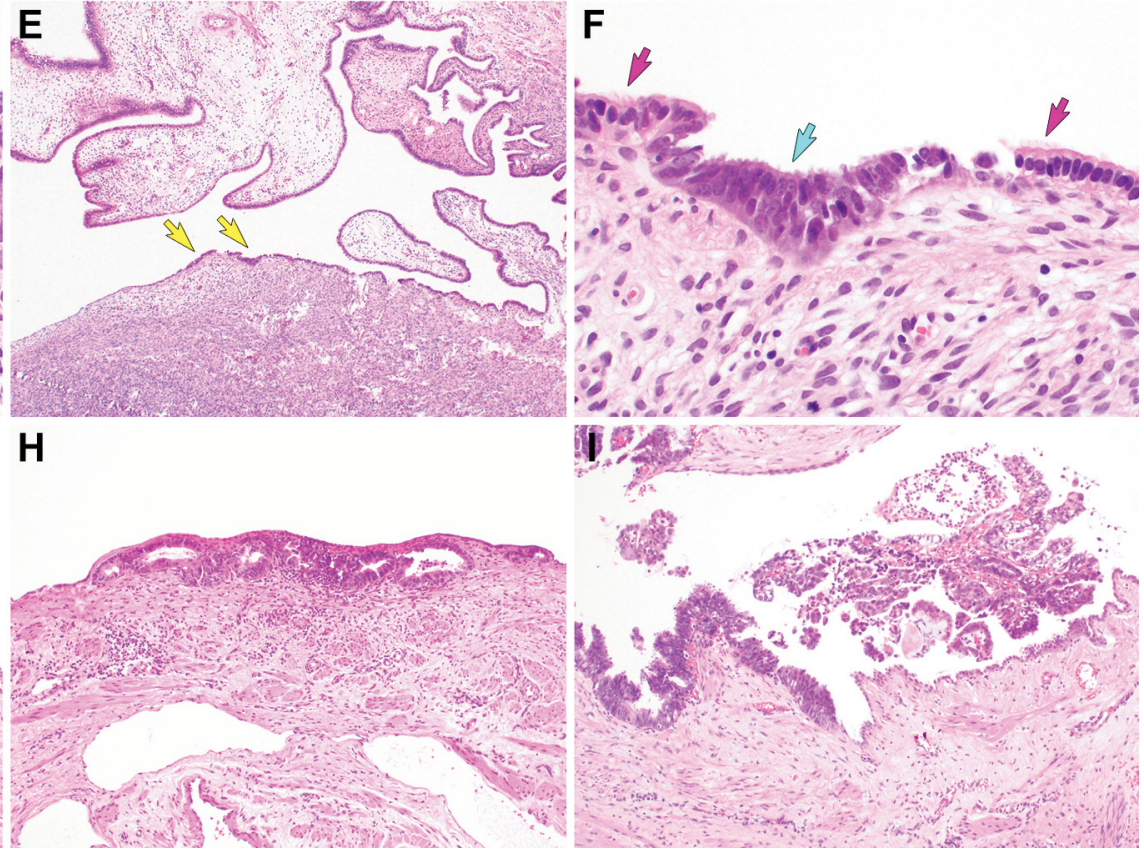

Figure 2. Histological features of the fimbrial lesion observed in the initial microscopic examination. A: Low-power magnification revealed that the tip and edge of the fimbrial plica exhibited some microscopic foci of epithelial thickening (blue and green arrows), mimicking serous tubal intraepithelial carcinoma (STIC). B: The adjacent normal tubal epithelium (purple arrow) was arranged as a single layer of ciliated low-columnar cells, whereas the thickened epithelium displayed nuclear enlargement, hyperchromasia, stratification, irregular stacking, and loss of polarity. Apparent transition point was noted between the non-neoplastic tubal epithelium on the right and the neoplastic epithelium on the left. C: The neoplastic epithelium also showed architectural complexity with small detached micropapillary clusters, fusion of the micropapillae, and focal cribriform appearance. The subepithelial stroma was unremarkable. D: In addition to the architectural abnormalities, severe nuclear pleomorphism, conspicuous nucleoli, increased mitotic activity, and occasional atypical mitoses (red circle) further supported the possible diagnosis of STIC. E: A partial replacement of the ovarian surface epithelium by neoplastic epithelium (yellow arrows) was identified near the site of tubo-ovarian adhesion (yellow arrows). F: Similar to image B, highpower view demonstrated an abrupt transition between the single-layered non-neoplastic ciliated epithelium (purple arrow) and the neoplastic epithelium. G-I: Several microscopic foci of peritoneal metastases were detected. They were characterized by relatively well-circumscribed tissue plaques that seemed to be plastered on the surface of the peritoneum. Their cytological features were the same as those of the fimbrial lesions. Coexisting ovarian invasive carcinoma and tubal intraepithelial carcinoma spreading horizontally on the fimbrial surface were morphologically compatible with the diagnosis of high-grade serous carcinoma and STIC. Original magnification: A: 10x; (B-C) 100x; D: 400x; E: 10x; F: 400x; and G-I: 40x.

intraluminal secretions were occasionally noted within the tubular and ductal structures (Figure 4A-D). These histological features suggested the possibility of MLA. The intraoperative washing cytology specimen showed variablesized papillary tufts and irregular-shaped three-dimensional cellular clusters (Figure 4E-G). High-grade nuclear atypia observed under high-power magnification seemed to be characteristic of HGSC (Figure 4H-K). However, the small tubular lumina (Figure $4 \mathrm{H}$ and I) and deeply stained substances (Figure 4J and $\mathrm{K}$ ), which were similar to those identified in hematoxylin and eosin-stained histology slides, supported the possibility of MLA rather than HGSC. 

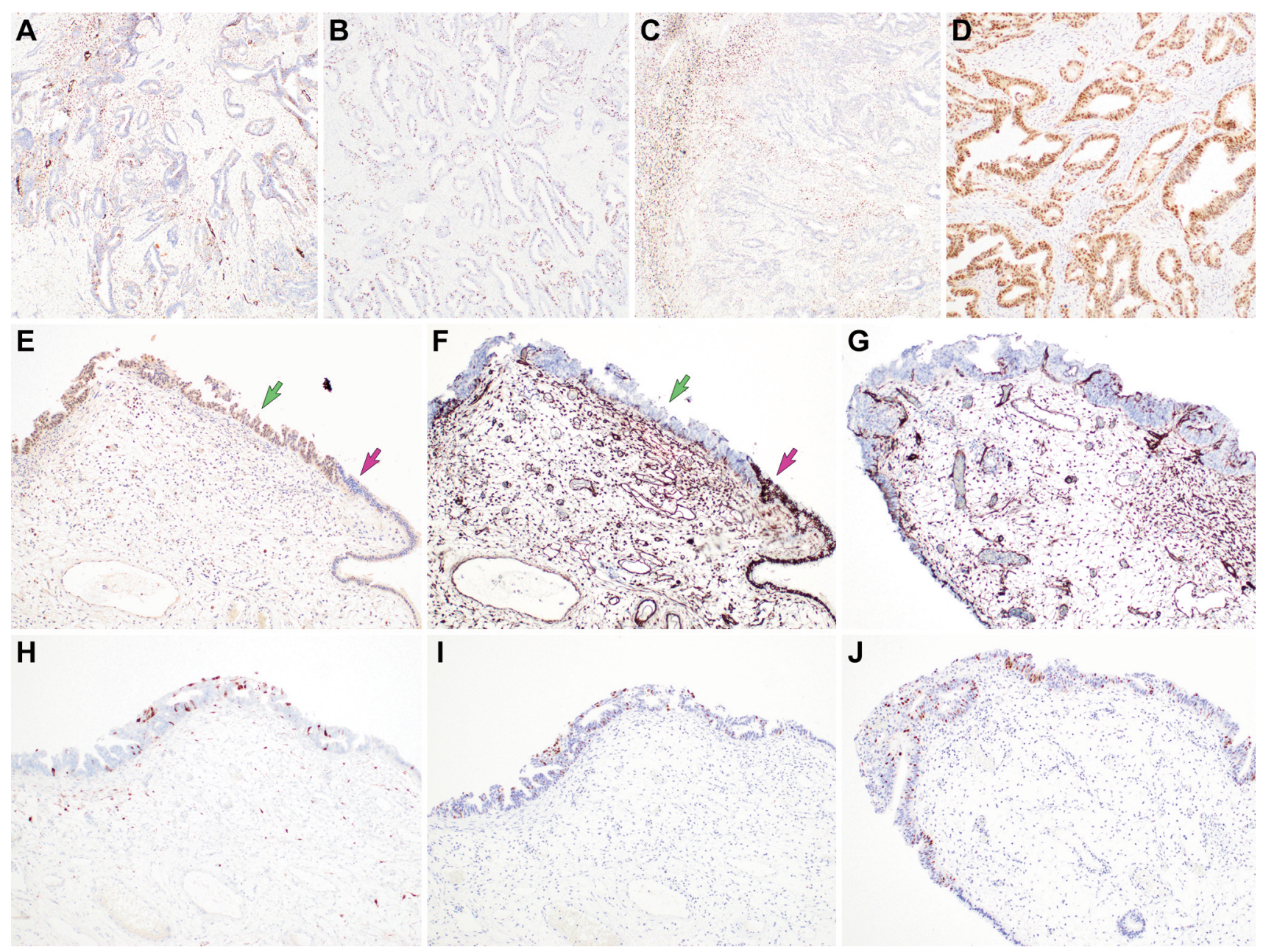

Figure 3. Immunostaining results using a polymer method. A: The ovarian tumor cells were completely negative for Wilms tumor 1 (WT1). B: Patchy nuclear p53 immunoreactivity with weak-to-moderate staining intensity, indicating wild-type p53 expression pattern. C: Expression of estrogen receptor was also completely absent. D: Instead, the ovarian tumor cells were diffusely and intensely positive for GATA-binding protein 3. E-G: The fimbrial tumors that were initially considered to be serous tubal intraepithelial carcinoma (STIC) displayed the same immunophenotype as that of the ovarian tumor. E: Paired box 2 was expressed in the tumor cells spreading along the fimbrial surface epithelium (green arrow) but not in the adjacent normal epithelium (purple arrow). F and G: The fimbrial tumor cells (green arrow) were negative for WT1, which highlighted the nuclei of normal tubal epithelium (purple arrow). H: Patchy p16 expression was observed within the tumor cell cytoplasm. I and J: Wild-type p53 expression pattern did not support the diagnosis of high-grade serous carcinoma and STIC. Original magnification: A-C: 40x; D: 100x; and E-J: 40x.

We performed additional immunostaining for progesterone receptor (PR), phosphatase and tensin homolog deleted on chromosome 10 (PTEN), mutL homolog 1 (MLH1), human postmeiotic segregation increased 2 (PMS2), mutS homolog 2 (MSH2), and mutS homolog 6 (MSH6). PR expression was not observed. The expression status of PAX2 and GATA3 was confirmed in the other tumor sections. No significant difference was observed in the intensity and proportion of PAX2 and GATA3 staining among the different tumor areas. PTEN, MLH1, PMS2, MSH2, and MSH6 were preserved in the cytoplasm of tumor cells. Targeted sequencing was also conducted, as previously described $(12,24,29-32)$. No pathogenic mutations were identified in the well-known hot spots of TP53 exons. No mutations affecting the splice sites or introns of TP53 were detected. Instead, both the ovarian and fimbrial tumors harbored the same pathogenic v-Ki-ras2 Kirsten rat sarcoma viral oncogene homolog (KRAS) mutation (c.35G>T; p.Gly12Val).

The patient received postoperative chemotherapy using a combination of paclitaxel-carboplatin. After the third treatment cycle (3 months postoperatively), abdominal pain occurred. Computed tomography revealed obstructive ileus with a distinct transition point in the terminal ileum. No 


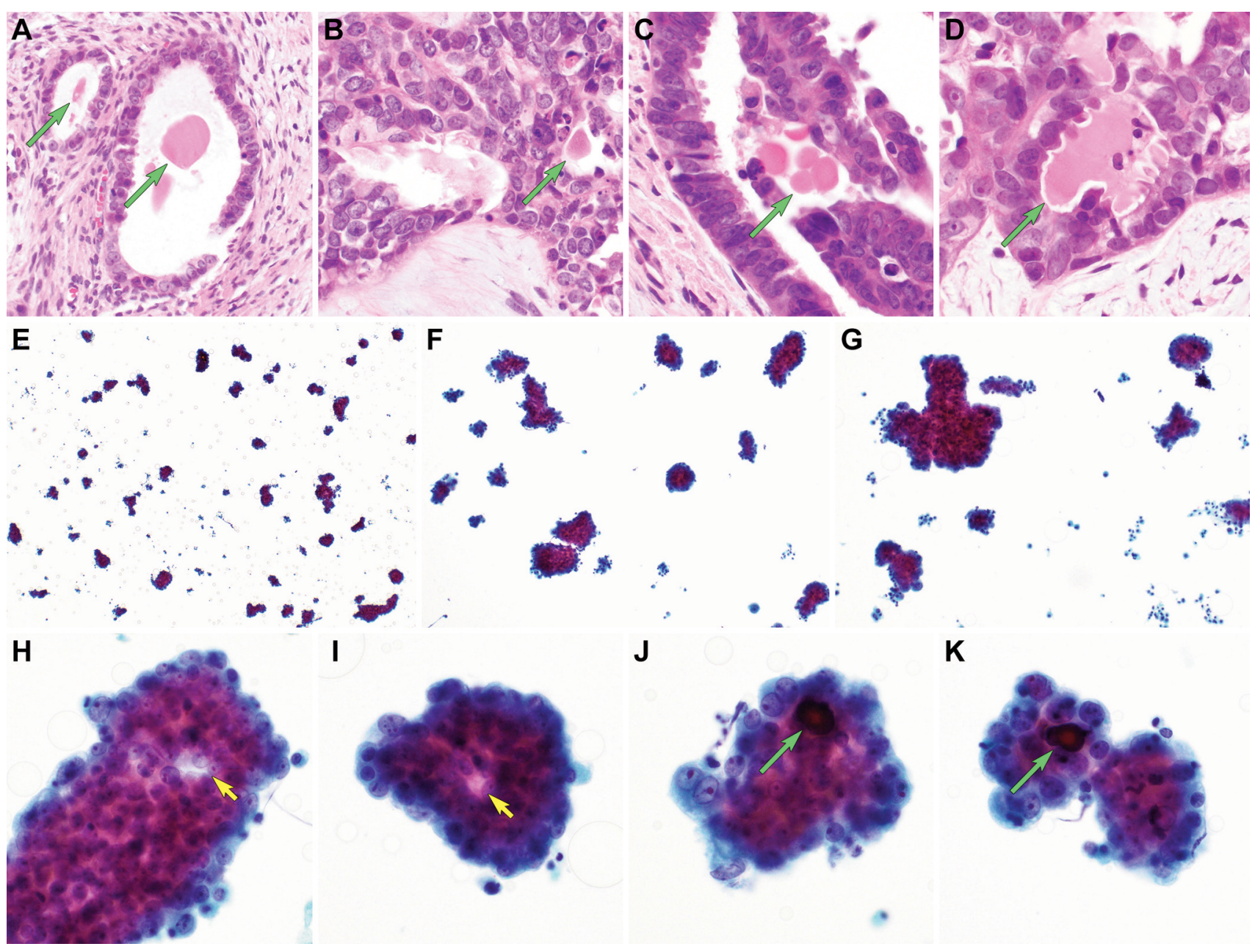

Figure 4. Histological (A-D) and cytological $(E-K)$ features noted during re-examination. A and B: Densely eosinophilic intraluminal secretions were occasionally identified in the tubules and glands (green arrows). They appeared as bright pink hyaline- or colloid-like substances, and were similar to those observed in benign mesonephric remnants or thyroid follicles. C and D: Moreover, they formed globules $(C)$ or conformed to the contours of the glands in which they were found (D, green arrows). E-G: Intraoperative washing cytology specimen revealed several papillary tufts and irregular-shaped three-dimensional cellular clusters. H-K: High-grade nuclear atypia observed in high-power magnification seemed characteristic of high-grade serous carcinoma. However, the presence of small tubular lumina (yellow arrows in $H$ and I) and deeply stained substances (green arrows in J and K) similar to those identified in the histological slides supported the possible diagnosis of mesonephric-like adenocarcinoma. Staining method: A-D: hematoxylin and eosin staining; E-K: Papanicolaou staining. Original magnification: A: 200x; B-D: 400x; E: 10x; F: 40x; G: 100x; H-I: 400x; and J-K: 600x.

measurable seeding lesions were observed. The patient completed six cycles of chemotherapy after the symptoms improved. At 11 months postoperatively, the patient is currently alive without any evidence of disease.

\section{Discussion}

We describe a case of ovarian MLA with multifocal microscopic involvement of the fimbrial surface. The patient presented with a 4.4-cm solid and cystic ovarian mass with multiple enlarged para-aortic lymph nodes. Histologically, the well-circumscribed ovarian tumor consisted of papillary and micropapillary growth patterns, solid sheets of tumor cells with slit-like spaces, and endometrioid-like glandular proliferation with cribriform architecture. The tumor cell nuclei demonstrated high-grade atypia and occasional mitosis. The tubal lesions consisted of atypical stratified epithelium horizontally spreading along the fimbrial surface and possessing hyperchromatic and pleomorphic nuclei. Their nuclei also showed loss of polarity, severe pleomorphism, increased mitotic activity, and atypical mitotic figures. These histological features prompted the 
diagnosis of HGSC accompanied by STIC. However, the complete absence of WT1 immunoreactivity and wild-type p53 expression pattern did not support the diagnosis of either HGSC or STIC. Instead, diverse architectural patterns and positive expressions of both mesonephric markers, PAX2 and GATA3, raised the possibility of MLA. A thorough microscopic review with additional immunostaining aided the final diagnosis of MLA based on the presence of small tubular aggregates with hyaline-like eosinophilic intraluminal secretions and lack of hormone receptor expression. Although the presence of severe nuclear pleomorphism and high mitotic rate were not compatible with the features of a typical MLA, we had previously found that these high-grade cytological features can be identified in a small subset of MLAs (32). The presence of ovarian and tubal lesions harboring pathogenic KRAS mutations, but not TP53 mutations, further confirmed the diagnosis of MLA.

STIC, which is observed in $60 \%$ of patients with tuboovarian and peritoneal HGSC (5), has been accepted as the earliest pathological manifestation of most HGSCs (33). The presence of occult, non-mass-forming $\operatorname{HGSCs}(34,35)$ or dysplastic epithelial changes (36) in the fallopian tubes of known BRCAl mutation carriers who underwent prophylactic bilateral salpingo-oophorectomy implies that the fallopian tubes are the anatomical site of origin of HGSC. Concurrent HGSC and STIC, incidentally discovered in patients not known to be at high risk, also provides further evidence supporting the tubal origin of HGSC. Gilks et al. reported that 21 incidental tubal HGSCs were accompanied by STIC and that most of them were confined to the fallopian tube (35). Moreover, according to the recently introduced criteria for assigning the origin of tubo-ovarian HGSC (37), the primary site should be assigned as being tubal if the STIC lesion or HGSC invades the fallopian tube, or when part or all of the tubes are inseparably incorporated within a tubo-ovarian mass. Detection of identical somatic TP53 mutations in STICs and concurrent HGSCs also supports the clonal relationship between the two lesions (38).

However, not all tubal intraepithelial carcinomas originate from the fallopian tube. Recent studies have documented that tubal IEMs of non-gynecological origin can mimic STIC. Rabban et al. investigated 100 patients with non-gynecological carcinomas that metastasized to the fallopian tubes (39). Approximately half of the patients had fimbrial involvement, and $29 \%$ had tubal mucosal involvement. The most common site of metastasis was the colorectum, followed by the upper gastrointestinal and pancreatobiliary tracts, appendix, and breasts. The growth patterns varied from flattened to stratified, exophytic, and pseudoinvasive. High-grade nuclear atypia was observed in approximately two-thirds of the patients with tubal mucosal growth. These tumors mimicked the morphological features of STIC or HGSC, including unilaterality, fimbrial location, severe nuclear pleomorphism, and increased mitotic activity. Reyes et al. also reported eight patients with human papillomavirus-associated endocervical adenocarcinoma with tubal metastasis (40). Seven out of the eight tumors were unilateral, while six had microscopically colonized within the tubal epithelium. These epithelial-limited lesions showed papillary tufting and slit-like spaces, simulating STIC and HGSC, and led to diagnostic confusion. However, the presence of stratified, tall, hyperchromatic nuclei with easily identifiable apical mitoses and apoptotic bodies, and positivity for human papillomavirus upon in situ hybridization support the diagnosis of metastatic endocervical adenocarcinoma. Similarly, we previously reported five patients with endometrial, cervical, and colorectal carcinomas with tubal IEMs (8). We found that the characteristic histological features of STIC, including cellular crowding with papillary configuration and nuclear stratification, loss of polarity, and high-grade nuclear atypia, were also observed in those with epithelial-limited metastasis, indicating that careful consideration of clinical history and the use of immunostaining are critical in making an accurate diagnosis. Taken together, not all tubal intraepithelial carcinomas are of tubal origin. Although uncommon, it is possible for metastases of both gynecological and nongynecological malignancies to grow within the mucosa of the fallopian tube and create a potential diagnostic pitfall. The intraepithelial growth of a tumor in the fallopian tube is not pathognomonic of the primary tubal origin of the tumor.

It is difficult to distinguish tubal IEM of ovarian MLAs from HGSC based on the morphological features alone. Destructive stromal invasion, papillary and solid architecture, severe nuclear pleomorphism throughout the entire tumor, and the presence of psammomatous microcalcifications are useful diagnostic features of HGSC. In contrast, the classic morphological pattern of MLA, that is, closely aggregated back-to-back tubules lined by cuboidal cells with low-tointermediate-grade nuclear atypia and containing densely eosinophilic intraluminal secretions, supports the diagnosis of MLA. Interestingly, this case showed overlapping morphological features of ovarian HGSC and MLA, which posed a diagnostic challenge. We previously demonstrated that severe nuclear pleomorphism with increased mitotic activity is an uncommon but possible histological presentation in a small subset of patients with MLAs (32). Conversely, HGSC can have eosinophilic intraluminal substances similar to those of MLA, but these are irregular, shattered materials that do not have the same shape as that of the lumen. MLAs have deeply eosinophilic, hyaline- or colloid-like secretions, which typically appear bright pink on hematoxylin and eosin staining and are similar to those observed in benign mesonephric lesions or thyroid follicles $(24,32)$. Moreover, the intraluminal secretions of MLA usually conform to the contours of the glands in which they are found. Nevertheless, intraluminal secretions are neither specific nor pathognomonic for HGSC or MLA. Given that 
both HGSC and MLA exhibit papillary and glandular architecture with high-grade nuclear atypia, they should be included in the differential diagnosis of uterine tumors exhibiting various growth patterns.

Immunostaining for a panel of antibodies can be useful in distinguishing MLA with tubal IEM from HGSC accompanied by STIC. Ovarian MLA shares an immunophenotype with cervical MA, which is different from that of ovarian HGSC (15, 41-43). The MLA cells show either complete negativity or focal to weak positivity for hormone receptors, lack of WT1 expression, or wild-type p53 immunostaining pattern, while being positive for mesonephric markers, PAX2 and GATA3 $(8,41,43)$. A previous study reported that ovarian MLAs exhibit nuclear immunoreactivity for thyroid transcription factor-1 (43). In contrast, HGSC typically reacts diffusely and intensely for WT1 protein. They exhibit aberrant p53 expression as well as positivity for ER and PR, with variable staining intensities and proportions. In our case, the tumor lacked immunoreactivity for WT1, ER and PR, and showed a wild-type p53 immunostaining pattern, which excluded the possibility of HGSC. The diffuse positivity for PAX2, a protein associated with the development of the Wolffian system, is typical of mesonephric tumors (41), and GATA3, the best marker for MA and MLA with high sensitivity and specificity (43), supported the diagnosis of MLA. In addition, pathogenic KRAS mutations were observed, but not TP53 mutations. For patients with high-grade ovarian carcinoma showing morphological features of both HGSC and MLA, targeted sequencing can help confirm the presence of characteristic mutations for each particular subtype.

In summary, we describe a rare case of ovarian MLA with multifocal microscopic fimbrial involvement. The invasive ovarian tumor exhibited dominant papillary and solid architecture and high-grade nuclear atypia, compatible with HGSC. The fimbria was infiltrated with tumor cells that spread along the surface epithelium, but did not invade the subepithelial stroma, and displayed the same cytological features as those of the ovarian lesion, compatible with STIC. Based on these histological features, this case was initially considered as ovarian HGSC associated with STIC. However, immunostaining results revealed that the ovarian and tubal lesions were negative for WT1 and ER, which are characteristic markers of HGSC and STIC, and positive for PAX2 and GATA3, which are mesonephric markers. In addition, targeted sequencing analysis revealed that the tumor harbored a pathogenic KRAS mutation, a characteristic genetic alteration of MLA, but none of the pathogenic TP53 mutations. Ovarian MLA can also show intraepithelial spread along the fimbrial surface; in this case, the features of MLA appeared very similar to those of STIC. Since MLA can present various architectural patterns, it can mimic HGSC, the most common ovarian carcinoma, especially when it presents as an ovarian tumor with papillary and solid architecture.
However, observation of the various growth patterns and the small tubules containing eosinophilic intraluminal secretions through a thorough microscopic examination can lead to the diagnosis of MLA. In addition, further verification can be done by performing additional immunostaining and targeted sequencing to confirm the immunophenotype and molecular features of MLA.

\section{Conflicts of Interest}

None of the Authors have any conflicts of interest or financial ties to declare regarding this study.

\section{Authors' Contributions}

All Authors made substantial contributions to the conception and design of the study; the acquisition, analysis, and interpretation of the data; drafting of the article; critical revision of the article for important intellectual content; and the final approval of the version to be published.

\section{Acknowledgements}

This study was supported by a grant from the National Research Foundation of Korea (NRF) grant funded by the Korean government (MSIT) (NRF-2019R1G1A1100578).

\section{References}

1 Bachert SE, McDowell A Jr, Piecoro D and Baldwin Branch L: Serous tubal intraepithelial carcinoma: a concise review for the practicing pathologist and clinician. Diagnostics (Basel) 10(2): 102, 2020. PMID: 32069831. DOI: 10.3390/diagnostics10020102

2 Mehrad M, Ning G, Chen EY, Mehra KK and Crum CP: A pathologist's road map to benign, precancerous, and malignant intraepithelial proliferations in the fallopian tube. Adv Anat Pathol 17(5): 293-302, 2010. PMID: 20733351. DOI: 10.1097/PAP.0b013e3181ecdee1

3 Stanciu PI, Ind TEJ, Barton DPJ, Butler JB, Vroobel KM, Attygalle AD and Nobbenhuis MAE: Development of peritoneal carcinoma in women diagnosed with Serous Tubal Intraepithelial Carcinoma (STIC) following Risk-Reducing SalpingoOophorectomy (RRSO). J Ovarian Res 12(1): 50, 2019. PMID: 31128592. DOI: 10.1186/s13048-019-0525-1

4 Meserve EEK, Brouwer J and Crum CP: Serous tubal intraepithelial neoplasia: the concept and its application. Mod Pathol 30(5): 710-721, 2017. PMID: 28106106. DOI: 10.1038/ modpathol.2016.238

5 WHO Classification of Tumors Editorial Board: WHO Classification of Tumours: Female Genital Tumours. IARC, Lyon, 2020.

6 Kezlarian B, Muller S, Werneck Krauss Silva V, Gonzalez C, Fix DJ, Park KJ and Murali R: Cytologic features of upper gynecologic tract adenocarcinomas exhibiting mesonephric-like differentiation. Cancer Cytopathol 127(8): 521-528, 2019. PMID: 31318491. DOI: 10.1002/cncy.22160

7 Pors J, Ho J, Prentice L, Thompson E, Cochrane D, Gibbard E, Huntsman D, Gilks B and Hoang LN: c-KIT analysis and 
targeted molecular sequencing of mesonephric carcinomas of the female genital tract. Am J Surg Pathol 44(4): 495-502, 2020. PMID: 31714258. DOI: 10.1097/PAS .0000000000001403

$8 \mathrm{Na} \mathrm{K}$ and Kim HS: Clinicopathological characteristics of fallopian tube metastases from primary endometrial, cervical, and nongynecological malignancies: a single institutional experience. Virchows Arch 471(3): 363-373, 2017. PMID: 28702779. DOI: $10.1007 / \mathrm{s} 00428-017-2186-\mathrm{z}$

9 Mirkovic J, Sholl LM, Garcia E, Lindeman N, MacConaill L, Hirsch M, Dal Cin P, Gorman M, Barletta JA, Nucci MR, McCluggage WG and Howitt BE: Targeted genomic profiling reveals recurrent KRAS mutations and gain of chromosome 1q in mesonephric carcinomas of the female genital tract. Mod Pathol 28(11): 1504-1514, 2015. PMID: 26336887. DOI: 10.1038/modpathol.2015.103

10 Mirkovic J, McFarland M, Garcia E, Sholl LM, Lindeman N, MacConaill L, Dong F, Hirsch M, Nucci MR, Quick CM, Crum $\mathrm{CP}$, McCluggage WG and Howitt BE: Targeted genomic profiling reveals recurrent KRAS mutations in mesonephric-like adenocarcinomas of the female genital tract. Am J Surg Pathol 42(2): 227-233, 2018. PMID: 28984674. DOI: 10.1097/PAS. 0000000000000958

11 Kolin DL, Costigan DC, Dong F, Nucci MR and Howitt BE: A combined morphologic and molecular approach to retrospectively identify KRAS-mutated mesonephric-like adenocarcinomas of the endometrium. Am J Surg Pathol 43(3): 389-398, 2019. PMID: 30489318. DOI: 10.1097/PAS.0000000000001193

$12 \mathrm{Kim}$ H, Yoon N, Woo HY, Lee EJ, DO SI, Na K and Kim HS: Atypical mesonephric hyperplasia of the uterus harbors pathogenic mutation of Kirsten Rat Sarcoma 2 viral oncogene homolog (KRAS) and gain of chromosome 1q. Cancer Genomics Proteomics 17(6): 813-826, 2020. PMID: 33099482. DOI: $10.21873 / \mathrm{cgp} .20235$

13 Howitt BE, Emori MM, Drapkin R, Gaspar C, Barletta JA, Nucci MR, McCluggage WG, Oliva E and Hirsch MS: GATA3 is a sensitive and specific marker of benign and malignant mesonephric lesions in the lower female genital tract. Am J Surg Pathol 39(10): 1411-1419, 2015. PMID: 26135559. DOI: 10.1097/PAS.0000000000000471

14 McFarland M, Quick CM and McCluggage WG: Hormone receptor-negative, thyroid transcription factor 1-positive uterine and ovarian adenocarcinomas: report of a series of mesonephriclike adenocarcinomas. Histopathology 68(7): 1013-1020, 2016. PMID: 26484981. DOI: 10.1111/his.12895

15 Na K, Kim HS, Shim HS, Chang JH, Kang SG and Kim SH: Targeted next-generation sequencing panel (TruSight Tumor 170) in diffuse glioma: a single institutional experience of 135 cases. J Neurooncol 142(3): 445-454, 2019. PMID: 30710203. DOI: 10.1007/s11060-019-03114-1

16 Kwon HJ, Song SY and Kim HS: Prominent papillary growth pattern and severe nuclear pleomorphism induced by neoadjuvant chemotherapy in ovarian mucinous carcinoma: potential for misdiagnosis as high-grade serous carcinoma. Anticancer Res 41(3): 1579-1586, 2021. PMID: 33788752. DOI: 10.21873/anticanres.14918

17 Park S, Cho EY, Oh YL, Park YH and Kim HS: Primary peritoneal high-grade serous carcinoma misinterpreted as metastatic breast carcinoma: a rare encounter in peritoneal fluid cytology. Anticancer Res 40(5): 2933-2939, 2020. PMID: 32366445. DOI: 10.21873 /anticanres.14271
18 Park CK, Kim YW, Koh HH, Yoon N, Bae GE and Kim HS: Clinicopathological characteristics of squamous cell carcinoma and high-grade squamous intraepithelial lesions involving endocervical polyps. In Vivo 34(5): 2613-2621, 2020. PMID: 32871791. DOI: 10.21873 /invivo.12079

19 Kim SW, Kim HS and Na K: Characterization of paired box 8 (PAX8)-expressing metastatic breast carcinoma. Anticancer Res 40(10): 5925-5932, 2020. PMID: 32988924. DOI: 10.21873/ anticanres. 14613

20 Kim SJ, Kim SW, Oh CH, Hong M, DO SI, Kim YW, Kim HS and $\mathrm{Na} \mathrm{K}$ : Expression of insulin-like growth factor II mRNAbinding protein 3 in gallbladder carcinoma. Anticancer Res 40(10): 5777-5785, 2020. PMID: 32988905. DOI: 10.21873/ anticanres.14594

21 Kim HS, DO SI, Kim DH and Apple S: Clinicopathological and prognostic significance of programmed death ligand 1 expression in Korean patients with triple-negative breast carcinoma. Anticancer Res 40(3): 1487-1494, 2020. PMID: 32132048. DOI: 10.21873/anticanres.14093

22 Choi S, Na K, Kim SW and Kim HS: Dedifferentiated mesonephric-like adenocarcinoma of the uterine corpus. Anticancer Res 41(5): 2719-2726, 2021. PMID: 33952503. DOI: 10.21873/anticanres.15053

23 Choi S, Joo JW, Do SI and Kim HS: Endometrium-limited metastasis of extragenital malignancies: a challenge in the diagnosis of endometrial curettage specimens. Diagnostics (Basel) 10(3): 150, 2020. PMID: 32164210. DOI: 10.3390/diagnostics 10030150

24 Choi S, Jung YY and Kim HS: Serous carcinoma of the endometrium with mesonephric-like differentiation initially misdiagnosed as uterine mesonephric-like adenocarcinoma: a case report with emphasis on the immunostaining and the identification of splice site TP53 mutation. Diagnostics (Basel) 11(4): 717, 2021. PMID: 33919505. DOI: 10.3390/diagnostics11040717

25 Choi S, Cho J, Lee SE, Baek CH, Kim YK, Kim HJ and Ko YH: Adenocarcinoma of the minor salivary gland with concurrent MAML2 and EWSR1 alterations. J Pathol Transl Med 55(2): 132138, 2021. PMID: 33472334. DOI: 10.4132/jptm.2020.12.11

26 Choi S, Park S, Chung MP, Kim TS, Cho JH and Han J: A rare case of adenosquamous carcinoma arising in the background of IgG4-related lung disease. J Pathol Transl Med 53(3): 188-191, 2019. PMID: 30853707. DOI: 10.4132/jptm.2019.02.21

27 Jang Y, Jung H, Kim HN, Seo Y, Alsharif E, Nam SJ, Kim SW, Lee JE, Park YH, Cho EY and Cho SY: Clinicopathologic characteristics of HER2-positive pure mucinous carcinoma of the breast. J Pathol Transl Med 54(1): 95-102, 2020. PMID: 31718120. DOI: $10.4132 /$ jptm.2019.10.24

$28 \mathrm{Kim} \mathrm{H}, \mathrm{Kim}$ J, Lee SK, Cho EY and Cho SY: TFE3-expressing perivascular epithelioid cell tumor of the breast. J Pathol Transl Med 53(1): 62-65, 2019. PMID: 30269476. DOI: 10.4132/jptm. 2018.08.30

29 Yoon N, Kim HS, Lee JW, Lee EJ, Maeng LS and Yoon WS: Targeted genomic sequencing reveals different evolutionary patterns between locally and distally recurrent glioblastomas. Cancer Genomics Proteomics 17(6): 803-812, 2020. PMID: 33099481. DOI: $10.21873 / \operatorname{cgp} .20234$

30 Jung H, Bae GE, Kim HM and Kim HS: Clinicopathological and molecular differences between gastric-type mucinous carcinoma and usual-type endocervical adenocarcinoma of the uterine cervix. Cancer Genomics Proteomics 17(5): 627-641, 2020. PMID: 32859641. DOI: 10.21873/cgp.20219 
31 Jung YY, Woo HY and Kim HS: Targeted genomic sequencing reveals novel TP53 in-frame deletion mutations leading to p53 overexpression in high-grade serous tubo-ovarian carcinoma. Anticancer Res 39(6): 2883-2889, 2019. PMID: 31177126. DOI: 10.21873/anticanres.13417

$32 \mathrm{Na} \mathrm{K}$ and Kim HS: Clinicopathologic and molecular characteristics of mesonephric adenocarcinoma arising from the uterine body. Am J Surg Pathol 43(1): 12-25, 2019. PMID: 29189288. DOI: 10.1097/PAS.0000000000000991

33 Gilbert L, Basso O, Sampalis J, Karp I, Martins C, Feng J, Piedimonte S, Quintal L, Ramanakumar AV, Takefman J, Grigorie MS, Artho G, Krishnamurthy S and DOvE Study Group: Assessment of symptomatic women for early diagnosis of ovarian cancer: results from the prospective DOvE pilot project. Lancet Oncol 13(3): 285-291, 2012. PMID: 22257524. DOI: $10.1016 /$ S1470-2045(11)70333-3

34 Paley PJ, Swisher EM, Garcia RL, Agoff SN, Greer BE, Peters KL and Goff BA: Occult cancer of the fallopian tube in BRCA1 germline mutation carriers at prophylactic oophorectomy: a case for recommending hysterectomy at surgical prophylaxis. Gynecol Oncol 8O(2): 176-180, 2001. PMID: 11161856. DOI: $10.1006 /$ gyno.2000.6071

35 Gilks CB, Irving J, Köbel M, Lee C, Singh N, Wilkinson N and McCluggage WG: Incidental nonuterine high-grade serous carcinomas arise in the fallopian tube in most cases: further evidence for the tubal origin of high-grade serous carcinomas. Am J Surg Pathol 39(3): 357-364, 2015. PMID: 25517954. DOI: 10.1097/PAS.0000000000000353

36 Piek JM, van Diest PJ, Zweemer RP, Jansen JW, Poort-Keesom RJ, Menko FH, Gille JJ, Jongsma AP, Pals G, Kenemans P and Verheijen RH: Dysplastic changes in prophylactically removed Fallopian tubes of women predisposed to developing ovarian cancer. J Pathol 195(4): 451-456, 2001. PMID: 11745677. DOI: $10.1002 /$ path. 1000

37 Singh N, Gilks CB, Hirschowitz L, Kehoe S, McNeish IA, Miller D, Naik R, Wilkinson N and McCluggage WG: Primary site assignment in tubo-ovarian high-grade serous carcinoma: Consensus statement on unifying practice worldwide. Gynecol Oncol 141(2): 195-198, 2016. PMID: 26827965. DOI: $10.1016 /$ j.ygyno.2015.10.022
38 Kuhn E, Kurman RJ, Vang R, Sehdev AS, Han G, Soslow R, Wang TL and Shih IeM: TP53 mutations in serous tubal intraepithelial carcinoma and concurrent pelvic high-grade serous carcinoma - evidence supporting the clonal relationship of the two lesions. J Pathol 226(3): 421-426, 2012. PMID: 21990067. DOI: 10.1002/path.3023

39 Rabban JT, Vohra P and Zaloudek CJ: Nongynecologic metastases to fallopian tube mucosa: a potential mimic of tubal high-grade serous carcinoma and benign tubal mucinous metaplasia or nonmucinous hyperplasia. Am J Surg Pathol 39(1): 35-51, 2015. PMID: 25025442. DOI: 10.1097/PAS.0000000000000293

40 Reyes C, Murali R and Park KJ: Secondary involvement of the adnexa and uterine corpus by carcinomas of the uterine cervix: a detailed morphologic description. Int J Gynecol Pathol 34(6): 551563, 2015. PMID: 26166722. DOI: 10.1097/PGP.00000000 00000206

41 Rabban JT, McAlhany S, Lerwill MF, Grenert JP and Zaloudek CJ: PAX2 distinguishes benign mesonephric and mullerian glandular lesions of the cervix from endocervical adenocarcinoma, including minimal deviation adenocarcinoma. Am J Surg Pathol 34(2): 137-146, 2010. PMID: 20061933. DOI: 10.1097/PAS.0b013e3181c89c98

42 Kenny SL, McBride HA, Jamison J and McCluggage WG: Mesonephric adenocarcinomas of the uterine cervix and corpus: HPV-negative neoplasms that are commonly PAX8, CA125, and HMGA2 positive and that may be immunoreactive with TTF1 and hepatocyte nuclear factor 1- $\beta$. Am J Surg Pathol 36(6): 799807, 2012. PMID: 22456609. DOI: 10.1097/PAS.0b013e31 $824 \mathrm{a} 72 \mathrm{c} 6$

43 Pors J, Cheng A, Leo JM, Kinloch MA, Gilks B and Hoang L: A Comparison of GATA3, TTF1, CD10, and calretinin in identifying mesonephric and mesonephric-like carcinomas of the gynecologic tract. Am J Surg Pathol 42(12): 1596-1606, 2018. PMID: 30148742 . DOI: 10.1097/PAS.0000000000001142

Received July 11, 2021

Revised August 11, 2021

Accepted August 26, 2021 